\title{
The Optimal Pain Management Methods Post Thoracic Surgery: A Literature Review
}

\author{
Christiana Bithas ${ }^{1}$, Andreas Papaleontiou ${ }^{1}$, Pawel Sokal ${ }^{1}$ and Amer Harky ${ }^{2 *}$ \\ ${ }^{1}$ School of Medicine, University of Liverpool, Liverpool, UK
}

${ }^{2}$ Department of Cardiothoracic surgery, Liverpool Heart and Chest, Liverpool, UK

*Corresponding author: Amer Harky, Department of Cardiothoracic surgery, Liverpool Heart and Chest, Liverpool, UK

\begin{abstract}
Post-operative pain control is one of the key factors that can aid in fast and safe recovery after any surgical interventions. Thoracic surgery can cause significant postoperative pain which can lead to delayed recovery, delayed hospital discharge and possibly increased risk of chest complications in the form of atelectasis and even lower respiratory infections. Therefore, appropriate pain management following thoracic surgery is mandatory to prevent development of such morbidities including chronic pain.
\end{abstract}

Keywords: Thoracic Surgery, Analgesia, VATS, Robotics, Thoracotomy

\section{Introduction}

Thoracic surgical procedures can result in severe pain which can present as a challenge to be appropriately managed postoperatively. In particular, thoracotomies are well known for their severity of pain due to the incision, manipulation of muscles and ligaments, retraction of the ribs with compression, stretching of the intercostal nerves, possible rib fractures, pleural irritation, and postoperative tube thoracotomy [1]. Recognition of this has contributed to the development of minimally invasive techniques such as video assisted thoracoscopic surgeries (VATS) and lately robotic surgery [1]. These techniques not only aim to produce better aesthetic results, but also reduce post-operative pain and enhance recovery without compromising the quality of treatment offered. Poor pain management can lead to several and serious complications such as lung atelectasis, hypostatic pneumonia due to avoidance of deep breathing in these patients as a result of pain and superimposed infection [1]. Pain management as a result, does not only lead to greater patient satisfaction, but it also reduces morbidity and mortality in patients undergoing thoracic surgery [2]. Historically, post-operative pain management for thoracic surgery involved the use of narcotics alongside parenteral or oral anti-inflammatory agents [2]. Post chest tube removal patients typically are transitioned to oral analgesia. Multiple additional pain control adjuncts were also implemented with differing levels of success [1]. Over time, intra-operative techniques have been developed which aims to target pain reduction postoperatively [2]. As our understanding of both pain management and the factors that play a role in the development of pain has increased, we have been able to target these and improve postoperative pulmonary morbidity and pain scores [1,2]. We aim to review different means of pain control in this paper in order to assess their effectiveness in achieving optimum results.

\section{Thoracotomy}

The mechanism of pain in thoracotomy involves the innervation of the intercostal, sympathetic, vagus and phrenic nerves [3]. Additionally, shoulder pain may result from stretching of the joints during the operation.

After a thoracotomy, pain can persist for two months or more, and in certain incidences it recurs after a period of cessation. The incidence of chronic pain post thoracotomy is reported to be $22-67 \%$ in the population [4]. Good surgical technique and effective acute post-operative pain treatment are evident means of preventing post-thoracotomy pain and consequent pulmonary complications [4]. Due to the multifactorial character of the pain, 
a multimodal approach to target pain is advised. Typically, both regional and systemic anaesthesia are administered. A combination of opioids such as fentanyl or morphine are typically used [5]. A variety of techniques for the administration of local anaesthetics are available at present, and the effectiveness of each is assessed in this paper.

\section{a) Thoracic Epidural Analgesia (TEA)}

TEA was the most widely used method of means of analgesia. It was the gold standard means of pain relief $[6,7]$. It is typically inserted prior to general anaesthesia, at the level of T5-T6, midway along the dermatomal distribution of the thoracotomy incision. A study by Tiippana et al. [8] measured the visual analogue scale (VAS) in order to assess the presence of pain during rest and at the time at which they coughed in 114 patients of whom 89 had TEA and 22 who had other methods of pain control. TEA was effective in alleviating pain at rest and during coughing. In TEA patients, the incidence of chronic pain of at least moderate severity was $11 \%$ and $12 \%$ at 3 and 6 months, respectively. The study found that at one week after discharge, $92 \%$ of all patients needed daily pain medication. The study advised for extended postoperative analgesia for up to the week post-discharge to be administered in order to manage this. The study however concluded overall, that TEA was effective in controlling evoked post-operative pain. However, the study did encounter problems of technical form in $24 \%$ of the epidural catheters. The incidence of chronic pain, however, was lower compared with previous studies where TEA was not used. Several other studies support that TEA is superior to less invasive methods. According to Shelley B. et al. [9] TEA was preferred by $62 \%$ of the respondents over paravertebral block (PVB) with $30 \%$ and other analgesic techniques with $8 \%$. Limitations of this technique included hypotension and urinary retention. Certain patients with active infection and on anticoagulation are excluded from epidural placement.

\section{b) Paravertebral Block (PVB)}

PVB is considered an effective method for pain management and its use has been increased in the recent years. This technique involves injecting local anaesthetic into the paravertebral space and it is able to block unilateral multi-segmental spinal and sympathetic nerves. Previous studies have shown that it is effective in achieving analgesia and is associated with a lower incidence of side effects such as nausea, vomiting, hypotension and urinary retention $[10,11]$. As the lungs are collapsed, it is associated with a lower risk of pneumothorax.

In a study by Davies R.G. et al. [10] there was no significant difference in pain scores, morphine consumption and supplementary use of analgesia between TEA and PVB. The rate of failed technique was lower in PVB (OR =0.28, $\mathrm{p}=0.007)$. Respiratory function was improved at both 24 and 48 hours with PVB but only significantly improved at 24 hours. c) Intercostal Nerve Block (ICNB)

ICNBs are generally administered as single injections at least two dermatomes above and below the thoracotomy incision [12]. It is performed percutaneously or under direct vision, using single injections or through placement of an intercostal catheter. It can also be formed using cryotherapy. It is associated with reduced post-operative pain scores; however, it is less effective than TEA in controlling chronic pain [12]. This was illustrated by a study by Sanjay et al. [12] which found that patients that underwent ICNB had higher pain scores 4 hours post-operatively, than those who received epidural anaesthesia using $0.25 \%$ bupivacaine $(\mathrm{p}<0.05)$. The study concluded that in the early post-operative period there was significant impact in pain relief for both techniques, but thereafter, epidural anaesthesia was proven to significantly reduce post thoracotomy pain over ICNB. Due to the multifactorial nature of post-thoracotomy pain, various approaches are required in order to target pain. ICNBs are useful in the blockade of intercostal nerves, whilst PVB and TEA appear to block the intercostal and sympathetic nerves. Due to the inability of regional anaesthesia to block the vagus and phrenic nerves which are implicated in the pathophysiology of pain, NSAIDs and opioids are required as adjuncts. TEA is proven to be the most effective means of treating pain alongside PVB; however, it is associated with more side effects than PVB. At present, there are a limited number of studies directly comparing pain control and post-operative outcomes between PVB and TEA. There is no conclusive evidence that either method is superior to the other regarding pain control.

\section{Video-Assisted Thoracoscopic Surgery (VATS)}

Existing evidence supports the noninferiority of thoracic PVB when compared to TEA for postoperative analgesia [13]. PVB is versatile and may be applied both unilaterally or bilaterally. It can be used to avoid contralateral sympathectomy, consequently minimising hypotension. This is an apparent advantage it has over thoracic epidural. Furthermore, it offers a more favourable side effect profile when compared to epidural anaesthesia. At present, the factors taken into consideration when selecting a regional technique include tolerance of side effects associated with TEA, consensus on best practice/technique, and operator experience [13]. A randomised controlled trial by Kosiński et al. [14] compared the analgesic efficacy of continuous thoracic epidural block and percutaneous continuous PVB in 51 patients undergoing VATS lobectomy. The primary outcome measures were postoperative static (at rest) and dynamic (coughing) visual analogue pain scores (VAS), patient-controlled morphine use and side-effect profile. The study found that pain control (VAS) was superior in the PVB group at 24 hours, both at rest (1.7 vs3.3, p=0.01) and on coughing (5.8 vs $6.6, p=0.023$ ), and control of pain at rest was also superior in the PVB group at 36 hours ( 3.0 vs $3.7(p=0.025)$ and at 48 hours (1.2 vs $2.0, p=0.026)$. There were no significant differences in the postoperative morphine requirements. In regard to side-effect 
profile, the study showed that the incidence of postoperative urinary retention (defined as no spontaneous micturition for 8 hours or ultrasound-assessed volume of the urinary bladder $>500 \mathrm{ml}$ ) was greater in the epidural group (64.0\% vs $34.6 \%, \mathrm{p}=0.0036)$, as was the incidence of hypotension $(32.0 \%$ vs $7.7 \%, \mathrm{p}=0.0031)$. There was no significant difference in the incidence of atelectasis ( $4.0 \%$ vs $7.7 \%, p=0.0542$ ). However, the incidence of pneumonia was significantly more frequent in the PVB group $(3.8 \%$ vs $0 \%$, $\mathrm{p}=0 / 0331$ ). Kosiński et al. concluded that PVB is as effective as thoracic epidural block in regard to pain management as it offers a superior safety profile with minimal postoperative complications. A further randomised controlled trial by Okajima et al. [15] compared the requirements for postoperative supplemental analgesia in 90 patients who received wither a PVB or thoracic epidural infusion for VATS lobectomy, segmentectomy or wedge resection. The main outcome measures were pain scores at rest (verbal rating scale $0=$ none and $10=$ maximum pain), blood pressure, side effects and overall satisfaction scores relating to pain control (1=dissatisfied and 5 =satisfied). The study found a similar frequency of supplemental analgesia $50 \mathrm{mg}$ diclofenac sodium suppository or $15 \mathrm{mg}$ pentazocine intramuscularly) for moderate pain in both groups, with $56 \%$ of those in the PVB group requiring $\geq 2$ doses, compared to $48 \%$ in the epidural group $(\mathrm{p}=0.26)$. Hypotension, defined as a systolic blood pressure $<90 \mathrm{mmHg}$, occurred more frequently in the epidural group $(21.2 \%$ vs $2.8 \%, \mathrm{p}=0.02)$. There was no difference in the incidence of pruritus (3.0\% vs $0 \%, \mathrm{p}=0.29)$ and post-operative nausea and vomiting ( $30.3 \%$ vs $25.0 \%, p=0.62$ ) between both groups. The study found no statistical difference between patient-reported satisfaction in pain control between epidural and PVB using the verbal rating scale (5.0 vs $4.5, \mathrm{p}=0.36$ ). The study concluded that PVB offered additional to equivalent analgesia to epidural, a lower incidence of haemodynamic instability postoperatively. A further study by Khoshbin et al. [16] performed an analysis on 81 patients undergoing VATS for pleural aspiration +/- pleurodesis, lung biopsies or bullectomy. The main outcome was postoperative pain levels, documented every 6 hours and scored against the Visual analogue Scale $(0=$ no pain, $10=$ worst possible pain). In both PVB and epidural groups, bupivacaine $0.125 \%$ was the local anaesthetic of choice, with clonidine added to the epidural infusion at $300 \mu \mathrm{g}$ in $500 \mathrm{ml}$. The study showed that there was no significant difference in mean pain scores between PVB or EP (2.1 vs 2.9, p=0.899), therefore concluding that PVB is as effective as epidural in controlling pain post-VATS.

\section{Robotic Lung Surgery}

Minimally invasive techniques are considered advantageous over open surgical approaches due to their shorter recovery times, reduced perceived levels of pain post-operatively and shorter postoperative length of stay in hospital [17-19]. Robotic surgery has become a popular method in recent years. Debate remains regarding whether robotic surgery is superior to VATS in regard with pain reduction. A case control study by Louie et al. [19] compared 45 robotic assisted lobectomies (RAL) to 34 VATS lobectomies. The study showed that both groups had a similar mean ICU stay ( 0.9 vs 0.6 days) and a mean total length of stay ( 4.0 vs 4.5 days). The study showed that patients that underwent robotic lobectomies had a shorter duration of analgesic use post-operatively $(p=0.039)$ and a shorter time resuming to normal everyday activities $(p=0.001)$. A limitation in this study was an inaccurate record of the amount of pain relief used by the patients, ultimately working as a confounding factor when interpreting the results. In a separate study by Jang et al. [18] 40 patients undergoing RAL were compared retrospectively to 80 VATS patients ( 40 initial patients and 40 most recent patients), all with resectable non-small cell lung cancer. The study showed that the post-operative median length of stay was significantly shorter in RAL patients compared to the initial VATS patients. The rate of post-operative complications was significantly lower in the RAL group (10\%) compared to the initial VATS group (32.5\%) and similar to the recent VATS group (17.5\%). Post-operative recovery was easier for patients in both the RAL and VATS group due to earlier mobilisation, allowing them to return to their everyday activities quicker. In a retrospective review by Kwon et al. [17] 74 patients undergoing robotic surgery, 227 patients undergoing VATS and 201 patients undergoing anatomical pulmonary resection were assessed and compared with regard to acute (visual pain score) and chronic pain (Pain DETECT questionnaire). The study showed that there was no significant difference in acute or chronic pain between patients undergoing robotic assisted surgery and VATS. Despite no significant difference in pain scores, $69.2 \%$ of patients who underwent robotic-assisted surgery felt the approach affected their pain versus $44.2 \%$ of the patients who underwent VATS ( $p=0.0330$ ). These results all support the superiority of robotic surgery over VATS and open approaches with regard to pain, length of hospital stay and recovery times. Both robotic surgery and VATS have their benefits i.e. two-versus three-dimensional view, instrument manoeuvrability, and reduced post-operative pain.

\section{Conclusion}

Since post-thoracotomy pain is multifactorial, a multimodal approach is required. In particular, ICNB blocks the intercostal nerves, and PVB and TEA appear to block the intercostal and sympathetic nerves. NSAIDs and opioids are required as valgus and phrenic nerve cannot be blocked by regional anaesthesia. TEA is evident to be the most effective in treating pain alongside with PVB. It is however associated with more side effects than PVB.

\section{References}

1. Sparks A, Stewart JR (2018) Review of Pain Management in Thoracic Surgery Patients, 2018. J Anesth Clin Res 9(4): 1-3.

2. Alzahrani T (2013) Pain relief following thoracic surgical procedures: A literature review of the uncommon techniques. Saudi J Anaesth 11(3): 327-331.

3. Gottschalk A, Cohen SP, Yang S, Ochroch EA (2006) Preventing and Treating Pain after Thoracic Surgery. Anesthesiology 104: 594-600. 
4. Sengupta S (2015) Post-operative pulmonary complications after thoracotomy. Indian J Anaesth 59(9): 618-626.

5. Mesbah A, Yeung J, Gao F (2016) Pain after thoracotomy. BJA Educ 16(1) $1-7$.

6. Sandler AN (1999) Post-thoracotomy analgesia and perioperative outcome. Minerva Anestesiol 65(5): 267-274.

7. Eng J, Sabanathan S (1993) Post-thoracotomy analgesia. J R Coll Surg Edinb 38(2): 62-68.

8. Tiippana E, Nilsson E, Kalso E (2003) Post-thoracotomy pain after thoracic epidural analgesia: a prospective follow-up study. Acta Anaesthesiol Scand 47(4): 433-438.

9. Shelley B, Macfie A, Kinsella (2011) Anesthesia for Thoracic Surgery: A Survey of UK Practice. J Cardiothorac Vasc Anesth 25(6): 1014-1017.

10. Davies RG, Myles PS, Graham JM (2006) A comparison of the analgesic efficacy and side-effects of paravertebral vs epidural blockade for thoracotomy-a systematic review and meta-analysis of randomized trials. Br J Anaesth 96(4): 418-426.

11. Joshi GP, Bonnet F, Shah R, Wilkinson RC, Camu F, et al. (2008) A Systematic Review of Randomized Trials Evaluating Regional Techniques for Postthoracotomy Analgesia. Anesth Analg 107(3): 1026-1040.

12. Sanjay O, Prashanth P, Tauro D (2003) Intercostal nerve blockade versus thoracic epidural analgesia for post thoracotomy pain relief. Indian J Thorac Cardiovasc Surg 19(3): 141-144.

13. Harky A, Clarke CG, Kar A, Bashir M (2019) Epidural analgesia versus paravertebral block in video-assisted thoracoscopic surgery. Interact Cardiovasc Thorac Surg 28(3): 404-406.
14. Kosiński S, Fryźlewicz E, Wiłkojć M, Ćmiel A, Zieliński M (2016) Comparison of continuous epidural block and continuous paravertebral block in postoperative analgaesia after video-assisted thoracoscopic surgery lobectomy: a randomised, non-inferiority trial. Anaesthesiol Intensive Ther 48(5): 280-287.

15. Okajima H, Tanaka O, Ushio M, Higuchi Y, Nagai Y, et al. (2015) Ultrasoundguided continuous thoracic paravertebral block provides comparable analgesia and fewer episodes of hypotension than continuous epidural block after lung surgery. J Anesth 29(3): 373-378.

16. Khoshbin E, Al Jilaihawi AN, Scott NB, Prakash D, Kirk AJB. (2011) An Audit of Pain Control Pathways Following Video-Assisted Thoracoscopic Surgery. Innov Technol Tech Cardiothorac Vasc Surg 6(4): 248-252.

17. Kwon ST, Zhao L, Reddy RM, Chang AC, Orringer MB, et al. (2017) Evaluation of acute and chronic pain outcomes after robotic, videoassisted thoracoscopic surgery, or open anatomic pulmonary resection. J Thorac Cardiovasc Surg 154(2): 652-659.

18. Jang HJ, Lee HS, Park SY, Zo JI (2011) Comparison of the Early RobotAssisted Lobectomy Experience to Video-Assisted Thoracic Surgery Lobectomy for Lung Cancer. Innov Technol Tech Cardiothorac Vasc Surg 6(5): 305-310.

19. Louie BE, Farivar AS, Aye RW, Vallières E (2012) Early Experience with Robotic Lung Resection Results in Similar Operative Outcomes and Morbidity When Compared with Matched Video-Assisted Thoracoscopic Surgery Cases. Ann Thorac Surg 93(5): 1598- 1605.
To Submit Your Article Click Here: Submit Article

DOI: $10.32474 /$ SCSOAJ.2019.03.000151

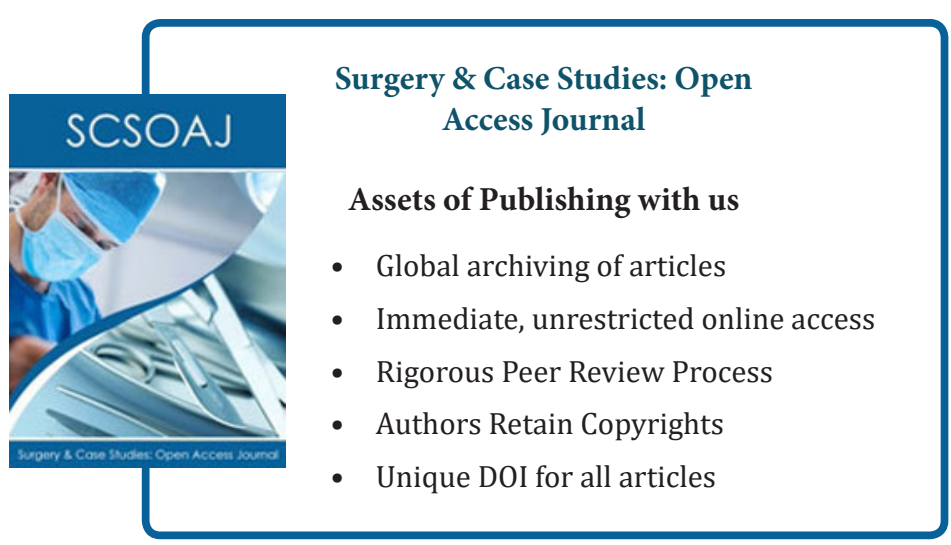

